\title{
Allergic Reaction, CTCAE
}

National Cancer Institute

\section{Source}

National Cancer Institute. Allergic Reaction, CT CAE. NCI Thesaurus. Code C143271.

A disorder characterized by an adverse local or general response from exposure to an allergen. 